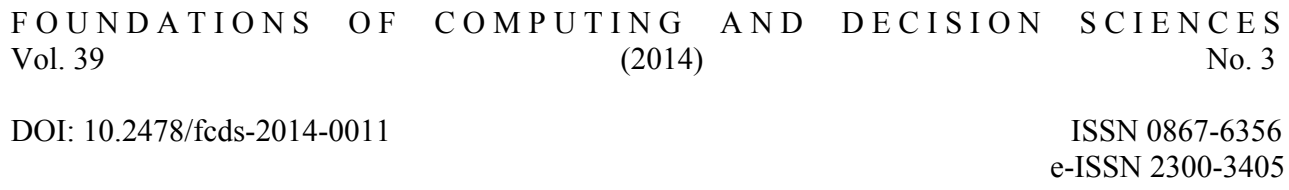

\title{
AGGREGATION OPERATORS ON TRIANGULAR INTUITIONISTIC FUZZY NUMBERS AND ITS APPLICATION TO MULTI-CRITERIA DECISION MAKING PROBLEMS
}

\author{
Changyong LIANG, Shuping ZHAO, Junling ZHANG*
}

\begin{abstract}
The aim of this work is to present some aggregation operators with triangular intuitionistic fuzzy numbers and study their desirable properties. Firstly, the score function and the accuracy function of triangular intuitionistic fuzzy number are given, the method for ranking triangular intuitionistic fuzzy numbers are developed. Then, some geometric aggregation operators for aggregating triangular intuitionistic fuzzy numbers are developed, such as triangular intuitionistic fuzzy weighted geometric (TIFWG) operator, the triangular intuitionistic fuzzy ordered weighted geometric (TIFOWG) operator and the triangular intuitionistic fuzzy hybrid geometric (TIFHG) operator. Moreover, an application of the new approach to multi-criteria decision making method was proposed based on the geometric average operator of TIFNs, and the new ranking method for TIFNs is used to rank the alternatives. Finally, an example analysis is given to verify and demonstrate the practicality and effectiveness of the proposed method.
\end{abstract}

Keywords: multi-criteria decision making, triangular intuitionistic fuzzy numbers, aggregation operation

\section{Introduction}

Since the influences of subjective and objective factors, it is not easy for decision makers to give the accurate evaluation on complex things in practical decision problems. There usually exist some hesitations for decision makers to assess the fuzzy and uncertain quantities. Therefore, Atanassov[6] introduced the concept of intuitionistic fuzzy set (IFS) characterized by a membership function and a non-membership function, which is a generalization of the concept of fuzzy set[40]. The IFS has been proven to be highly useful

${ }^{*}$ Changyong Liang ${ }^{1,2}$, Shuping Zhao ${ }^{1,2}(\bowtie)$, Junling Zhang ${ }^{3}$

1. School of Management, Hefei University of Technology, Hefei230009, China.

2. Key Laboratory of Process Optimization and Intelligent Decision-Making, Ministry of Education, Hefei 230009, China

3.School of Economics and Management, Zhejiang Normal University, Jinhua 321004, China

E-mail: zhaoshuping1753@163.com. E-mail: zhangjunling_dc@126.com. 
to deal with uncertainty and vagueness. Later, Atanassov and Gargov[15] further introduced the interval-valued intuitionistic fuzzy set (IVIFS), which is a generalization of intuitionistic fuzzy sets and fuzzy set. The intuitionistic fuzzy set and interval-valued intuitionistic fuzzy set were studied by many researchers[1, 5, 10], such as operators[7, 36, $38]$, operations $[2,4,8]$ and distances [3, 24], and have been applied to many different fields, such as decision making[12, 13, 23, 29, 37], supplier selection[9, 41], investment option[32, 33]et.al.

However, the domain of intuitionistic fuzzy set and interval-valued intuitionistic fuzzy set are discrete sets, therefore they are only used to indicate the extent to which the criterion does or does not belong to some fuzzy concepts[25]. To overcome this flaw, Shu et al.[22] gave the definition and operational laws of triangular intuitionistic fuzzy number. A prominent characteristic of the triangular intuitionistic fuzzy set is that its domain is a consecutive set. Some authors had paid attention to the research on triangular intuitionistic fuzzy numbers $[16,17,20,21,26]$, these researches can be roughly classified into two types: decision making methods $[11,17,18,20,26,27]$ and aggregation operators, which are respectively reviewed as follows. In the aspect of decision making methods, $\mathrm{Li}$ [16]pointed out and corrected some errors in the definition of the operational laws of triangular intuitionistic fuzzy numbers introduced by Shu et al.[22]. Li[17] discussed the concept of triangular intuitionistic fuzzy number and the ranking method of triangular intuitionistic fuzzy number on the basis of the concept of a ratio of the value index to the ambiguity index as well as applications to MADM problems. Nan et al. [20]defined the ranking order relations of triangular intuitionistic fuzzy number, which are applied to matrix games with payoffs of triangular intuitionistic fuzzy number. Wan[26]introduced the notions of possibility mean and variance for triangular intuitionistic fuzzy numbers, developed a new decision method based on possibility mean and variance of triangular intuitionistic fuzzy numbers. Li et al[18] defined the values and ambiguities of the membership degree and the non-membership degree for triangular intuitionistic fuzzy number as well as the value-index and ambiguity-index, and developed a ranking method based on value and ambiguity. In the aspect of aggregation operators [21, 28], Robinson P. $\mathrm{J}$ [21] defined the triangular intuitionistic fuzzy ordered weighted averaging (TIFOWA) operator and the triangular intuitionistic fuzzy hybrid aggregation (TIFHA) operator, and an extended TOPSIS model is developed to solve the multiple attribute group decision making problems under triangular intuitionistic fuzzy sets by using its correlation coefficient. Wang[28] proposed new arithmetic operations and logic operators for triangular intuitionistic fuzzy numbers and applied them to fault analysis of a printed circuit board assembly system. Through the existing literature, we can found that the aggregation operators of triangular intuitionistic fuzzy numbers is still quite limited, and the methods for ranking triangular intuitionistic fuzzy numbers are a bit complicated, which are inconvenient to compare triangular intuitionistic fuzzy numbers.

Compared with intuitionistic fuzzy numbers(IFNs), triangular intuitionistic fuzzy numbers are defined by using triangular fuzzy numbers expressing their membership and non-membership functions, which makes the membership degrees and the non-membership degrees no longer relative to a fuzzy concept "Excellent" or "Good", but relative to the triangular fuzzy number; then, the information of decision makers can be reflected exactly and can be expressed in different dimensions[30]. Thus, the information of decision makers can be reflected exactly and can be expressed in different dimensions than IFNs. As the aggregation operators are critically important tools of information fusion in multiple 
criteria decision making (MCDM) problems. The aim of this paper is to present some aggregation operators of triangular intuitionistic fuzzy numbers and a new method for ranking triangular intuitionistic fuzzy numbers. Thereby, a new multi-criteria decision making method using triangular intuitionistic fuzzy number is then proposed based on geometric average operators of triangular intuitionistic fuzzy numbers.

In order to do that, this work is set out as follows. In Section 2, some basic concepts and operation laws related to triangular intuitionistic fuzzy numbers are introduced, and the distance of triangular intuitionistic fuzzy number is defined. In Section 3, the expected values, the score function and the accuracy function of triangular intuitionistic fuzzy number are given, and the ranking method is developed for triangular intuitionistic fuzzy numbers based on the score values and the accuracy function values. In Section 4, the concept of the triangular intuitionistic fuzzy weighted geometric (TIFWG) operator, the triangular intuitionistic fuzzy ordered weighted geometric (TIFOWG) operator and the triangular intuitionistic fuzzy hybrid geometric (TIFHG) operator are proposed and their desirable properties are studied. In Section 5, based on the ITFWG, TIFOWG and ITFHG operators, a new method to solve multi-criteria decision making problems with triangular intuitionistic fuzzy information is proposed. Finally, an illustrative example is given to verify the developed approach.

\section{Preliminaries}

We start this section by introducing some basic concepts related to triangular intuitionistic fuzzy numbers, which will be used throughout this paper. The concept of triangular intuitionistic fuzzy number and some operational laws of triangular intuitionistic fuzzy numbers as follows:

Definition $1[17,22]$. Let $\tilde{\alpha}$ is a triangular intuitionistic fuzzy number, whose membership function and non-membership function are defined as follows, its membership function is:

$$
u_{\tilde{\alpha}}(x)= \begin{cases}(x-a) u_{\tilde{\alpha}} /(b-a) & (a \leq x<b) \\ \left.u_{\tilde{\alpha}}-x\right) u_{\tilde{\alpha}} /(c-b) & (b<x \leq c) \\ (c) & \text { otherwise }\end{cases}
$$

its non-membership function is:

$$
v_{\tilde{\alpha}}(x)=\left\{\begin{array}{lc}
\left(b-x+v_{\tilde{\alpha}}\left(x-a^{\prime}\right)\right) /\left(b-a^{\prime}\right) & \left(a^{\prime} \leq x<b\right) \\
v_{\tilde{\alpha}} & (x=b) \\
\left(x-b+v_{\tilde{\alpha}}\left(c^{\prime}-x\right)\right) /\left(c^{\prime}-b\right) & \left(b<x \leq c^{\prime}\right) \\
0 & \text { otherwise }
\end{array}\right.
$$

where $0 \leq u_{\tilde{\alpha}} \leq 1 ; 0 \leq v_{\tilde{\alpha}} \leq 1 ; 0 \leq u_{\tilde{\alpha}}+v_{\tilde{\alpha}} \leq 1, a, b, c, a^{\prime}, \mathrm{c}^{\prime} \in \mathrm{R}$. The intuitionistic fuzzy number is denoted as $\tilde{\alpha}=\left\langle\left([a, b, c] ; u_{\tilde{\alpha}}\right),\left(\left[a^{\prime}, b, c^{\prime}\right] ; v_{\tilde{\alpha}}\right)\right\rangle, \quad \tilde{\alpha}$ is called triangular intuitionistic fuzzy number. When $u_{\tilde{\alpha}}=1$ and $v_{\tilde{\alpha}}=0, \tilde{\alpha}$ is changed into triangular fuzzy 
number. For convenience, let $\tilde{\alpha}=\left([a, b, c] ; u_{\tilde{\alpha}}, v_{\tilde{\alpha}}\right)$. Without special declaration in this article, triangular intuitionistic fuzzy numbers are all these fuzzy numbers. $\pi_{\tilde{\alpha}}(x)=1-u_{\tilde{\alpha}}(x)-v_{\tilde{\alpha}}(x)$ denotes the hesitation of fuzzy number, the smaller the $\pi_{\tilde{\alpha}}$, the more certain is the fuzzy number.

Definition 2[21]. Let $\tilde{\alpha}_{1}=\left(\left[a_{1}, b_{1}, c_{1}\right] ; u_{\tilde{\alpha}_{1}}, v_{\tilde{\alpha}_{1}}\right)$ and $\tilde{\alpha}_{2}=\left(\left[a_{2}, b_{2}, c_{2}\right] ; u_{\tilde{\alpha}_{2}}, v_{\tilde{\alpha}_{2}}\right)$ be two triangular intuitionistic fuzzy number, and $\lambda \geq 0$, then

1) $\tilde{\alpha}_{1}+\tilde{\alpha}_{2}=\left(\left[a_{1}+a_{2}, b_{1}+b_{2}, c_{1}+c_{2}\right] ; u_{\tilde{\alpha}_{1}}+u_{\tilde{\alpha}_{2}}-u_{\tilde{\alpha}_{1}} u_{\tilde{\alpha}_{2}}, v_{\tilde{\alpha}_{1}} v_{\tilde{\alpha}_{2}}\right)$;

2) $\tilde{\alpha}_{1} \tilde{\alpha}_{2}=\left(\left[a_{1} a_{2}, b_{1} b_{2}, c_{1} c_{2}\right] ; u_{\tilde{\alpha}_{1}} u_{\tilde{\alpha}_{2}}, v_{\tilde{\alpha}_{1}}+v_{\tilde{\alpha}_{2}}-v_{\tilde{\alpha}_{1}} v_{\tilde{\alpha}_{2}}\right)$;

3) $\lambda \tilde{\alpha}=\left([\lambda a, \lambda b, \lambda c] ; 1-\left(1-u_{\tilde{\alpha}}\right)^{\lambda},\left(v_{\tilde{\alpha}}\right)^{\lambda}\right), \lambda \geq 0$;

4) $\tilde{\alpha}^{\lambda}=\left(\left[a^{\lambda}, b^{\lambda}, c^{\lambda}\right] ;\left(u_{\tilde{\alpha}}\right)^{\lambda}, 1-\left(1-v_{\tilde{\alpha}}\right)^{\lambda}\right), \lambda \geq 0$;

In the following, we give the operational results by same laws in Definition 2 are also triangular intuitionistic fuzzy number.

Theorem 1. Let $\tilde{\alpha}_{1}=\left(\left[a_{1}, b_{1}, c_{1}\right] ; u_{\tilde{\alpha}_{1}}, v_{\tilde{\alpha}_{1}}\right)$ and $\tilde{\alpha}_{2}=\left(\left[a_{2}, b_{2}, c_{2}\right] ; u_{\tilde{\alpha}_{2}}, v_{\tilde{\alpha}_{2}}\right)$ be two triangular intuitionistic fuzzy number, then the operational laws between $\tilde{\alpha}_{1}$ and $\tilde{\alpha}_{2}$ are shown as follows:

(1) $\tilde{\alpha}_{1}+\tilde{\alpha}_{2}=\tilde{\alpha}_{2}+\tilde{\alpha}_{1}$,

(2) $\tilde{\alpha}_{1} \otimes \tilde{\alpha}_{2}=\tilde{\alpha}_{2} \otimes \tilde{\alpha}_{1}$,

(3) $\lambda\left(\tilde{\alpha}_{1}+\tilde{\alpha}_{2}\right)=\lambda \tilde{\alpha}_{1}+\lambda \tilde{\alpha}_{2} \quad \lambda \geq 0$,

(4) $\lambda_{1} \tilde{\alpha}+\lambda_{2} \tilde{\alpha}=\left(\lambda_{1}+\lambda_{2}\right) \tilde{\alpha} \quad \lambda_{1}, \lambda_{2} \geq 0$

(5) $\tilde{\alpha}^{\lambda_{1}} \otimes \tilde{\alpha}^{\lambda_{2}}=\tilde{\alpha}^{\lambda_{1}+\lambda_{2}} \quad \lambda_{1}, \lambda_{2} \geq 0$,

(6) $\tilde{\alpha}_{1}^{\lambda} \otimes \tilde{\alpha}_{2}^{\lambda}=\left(\tilde{\alpha}_{1} \otimes \tilde{\alpha}_{2}\right)^{\lambda} \lambda \geq 0$.

Definition 3. Let $\tilde{\alpha}_{1}=\left(\left[a_{1}, b_{1}, c_{1}\right] ; u_{\tilde{\alpha}_{1}}, v_{\tilde{\alpha}_{1}}\right)$ and $\tilde{\alpha}_{2}=\left(\left[a_{2}, b_{2}, c_{2}\right] ; u_{\tilde{\alpha}_{2}}, v_{\tilde{\alpha}_{2}}\right)$ are two triangular intuitionistic fuzzy number, then the normalized Hamming distance between $\tilde{\alpha}_{1}$ and $\tilde{\alpha}_{2}$ is defined as follows:

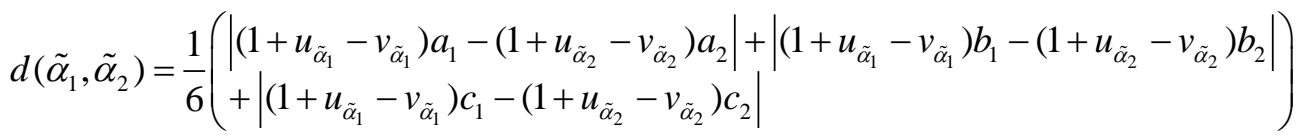

\section{A method for ranking triangular intuitionistic fuzzy numbers}

In this section, a method for ranking triangular intuitionistic fuzzy numbers is developed motivated by Wang' paper [14].The expected values, the score function and the accuracy function of triangular intuitionistic fuzzy numbers are defined as follows.

A useful tool for dealing with Fuzzy sets is their $\alpha$-cuts. Every $\alpha$-cut of a fuzzy number is a closed interval and a family of such intervals describes completely a fuzzy 
number under study.

Definition 4[17]. A $\alpha$-cuts set of triangular intuitionistic fuzzy number $\tilde{\alpha}=\left([a, b, c] ; u_{\tilde{\alpha}}, v_{\tilde{\alpha}}\right)$ is a crisp subset of $R$, which is defined as $\tilde{\alpha}_{\alpha}=\left\{x \mid u_{\tilde{\alpha}}(x) \geq \alpha\right\}$, where $0 \leq \alpha \leq u_{\tilde{\alpha}}$.

It directly follows from Eq. (1) and Definition 4 that $\tilde{\alpha}_{\alpha}=\left\{x \mid u_{\tilde{\alpha}}(x) \geq \alpha\right\}$ is a closed interval, denoted by $\tilde{\alpha}_{\alpha}=\left[L_{\tilde{\alpha}}(\alpha), R_{\tilde{\alpha}}(\alpha)\right]=\left[a+\frac{\alpha(b-a)}{u_{\tilde{\alpha}}}, c-\frac{\alpha(\mathrm{c}-b)}{u_{\tilde{\alpha}}}\right]$.

The trust degree of triangular intuitionistic fuzzy number $\tilde{\alpha}=\left([a, b, c] ; u_{\tilde{\alpha}}, v_{\tilde{\alpha}}\right)$ is between $\left[u_{\tilde{\alpha}}, 1-v_{\tilde{\alpha}}\right]$

Definition 5. Let $\tilde{\alpha}=\left([a, b, c] ; u_{\tilde{\alpha}}, v_{\tilde{\alpha}}\right)$ is a triangular intuitionistic fuzzy number, and then the expected value $E(\tilde{\alpha})$ of $\tilde{\alpha}$ is defined as follows:

$$
\begin{aligned}
& E_{\lambda}(\tilde{\alpha})=\frac{1}{2}\left(\int_{0}^{u_{\tilde{\alpha}}}\left\{(1-\lambda) \times L_{\tilde{\alpha}}(\alpha)+\lambda \times R_{\tilde{\alpha}}(\alpha)\right\} \mathrm{d} y+\int_{0}^{1-v_{\tilde{\alpha}}}\left\{(1-\lambda) \times L_{\tilde{\alpha}}(\alpha)+\lambda \times R_{\tilde{\alpha}}(\alpha)\right\} \mathrm{d} y\right) \\
& =\frac{1}{4}((1-\lambda)(a+b)+\lambda(b+c))\left(1+u_{\tilde{\alpha}}-v_{\tilde{\alpha}}\right)
\end{aligned}
$$

where $\lambda \in[0,1], \lambda$ varies according to the risk tolerance of the decision maker. The decision maker is risk-averse, if $\lambda<0.5$; The decision maker presents a propensity for risk, if $\lambda<0.5$; The decision maker is risk-neutral, if $\lambda=0.5$.

The score function and accuracy function of triangular intuitionistic fuzzy numbers are introduced below.

Definition 6. Let $\tilde{\alpha}=\left([a, b, c] ; u_{\tilde{\alpha}}, v_{\tilde{\alpha}}\right)$ is a triangular intuitionistic fuzzy number, and then the score function $S(\tilde{\alpha})$ and the accuracy function $H(\tilde{\alpha})$ of $\tilde{a}$ is defined as follows:

$$
\begin{gathered}
S(\tilde{\alpha})=E(\tilde{\alpha}) *\left(\mu_{\tilde{\alpha}}-v_{\tilde{\alpha}}\right) \\
H(\tilde{\alpha})=E(\tilde{\alpha}) *\left(\mu_{\tilde{a}}+v_{\tilde{a}}\right)
\end{gathered}
$$

There are several ranking methods $[17,26,27]$ of triangular intuitionistic fuzzy number which considers the DM's risk preference, but the calculation process is too complicated, so no unique best approach exists. Compared to the above method, the ranking method also consider the decision makers' risk preference, and the calculation process is simple. To facilitate the calculation, we assume that the decision maker is risk-neutral, the expected value $E(\tilde{\alpha})$ of $\tilde{a}$ is as follows in this paper

$$
E(\tilde{\alpha})=\frac{a+2 b+c}{4}\left(1+\mu_{\tilde{\alpha}}-v_{\tilde{\alpha}}\right)
$$

Definition 7. Let $\tilde{\alpha}_{1}$ and $\tilde{\alpha}_{2}$ are any two triangular intuitionistic fuzzy number, and then the

1) If $S\left(\tilde{\alpha}_{1}\right)>S\left(\tilde{\alpha}_{2}\right)$, then $\tilde{\alpha}_{1} \succ \tilde{\alpha}_{2}$;

2) If $S\left(\tilde{\alpha}_{1}\right)=S\left(\tilde{\alpha}_{2}\right)$, and $H\left(\tilde{\alpha}_{1}\right)=H\left(\tilde{\alpha}_{2}\right)$, then $\tilde{\alpha}_{1}=\tilde{\alpha}_{2}$; 
3) If $S\left(\tilde{\alpha}_{1}\right)=S\left(\tilde{\alpha}_{2}\right)$, and $H\left(\tilde{\alpha}_{1}\right)>H\left(\tilde{\alpha}_{2}\right)$, then $\tilde{\alpha}_{1} \succ \tilde{\alpha}_{2}$;

4) If $S\left(\tilde{\alpha}_{1}\right)=S\left(\tilde{\alpha}_{2}\right)$, and $H\left(\tilde{\alpha}_{1}\right)<H\left(\tilde{\alpha}_{2}\right)$, then $\tilde{\alpha}_{2} \succ \tilde{\alpha}_{1}$.

When the values of score function of the triangular intuitionistic fuzzy numbers are the same, the bigger the value of the accuracy function, the bigger the corresponding triangular intuitionistic fuzzy numbers.

\section{Some geometric aggregation operators on triangular intuitionistic fuzzy numbers}

In this section, we shall develop some geometric operators for aggregating triangular intuitionistic fuzzy numbers based on the literature[39]and study their desirable properties. Let $\Omega$ be the set of triangular intuitionistic fuzzy number. In the following, some aggregation operators with triangular intuitionistic fuzzy numbers are developed as follows:

Definition 8. Let $\tilde{\alpha}_{i}(i=1,2, \cdots, n)$ be a collection of triangular intuitionistic fuzzy number, and let TIFWG: $\Omega^{n} \rightarrow \Omega$, if

$$
\operatorname{TIFWG}\left(\tilde{\alpha}_{1}, \tilde{\alpha}_{2}, \cdots, \tilde{\alpha}_{n}\right)=\prod_{i=1}^{n} \tilde{\alpha}_{i}^{\omega_{i}}
$$

then TIFWG is called triangular intuitionistic fuzzy weighted geometric operator of dimension $n$, where $\omega=\left(\omega_{1}, \omega_{2}, \ldots, \omega_{n}\right)^{T}$ is the weight vector of $\tilde{\alpha}_{i}(i=1,2, \cdots, n)$, $\sum_{i=1}^{n} \omega_{i}=1, \omega_{i} \in[0,1]$. Especially, if $\omega=(1 / n, 1 / n, \ldots, 1 / n)^{T}$, the TIFWG operator is reduced to triangular intuitionistic fuzzy geometric averaging (TIFGA)operator, which is defined as follows:

$$
\operatorname{TIFGA}\left(\tilde{\alpha}_{1}, \tilde{\alpha}_{2}, \cdots, \tilde{\alpha}_{n}\right)=\left(\tilde{\alpha}_{1} \otimes \tilde{\alpha}_{2} \otimes \cdots \otimes \tilde{\alpha}_{n}\right)^{\frac{1}{n}}
$$

By Definition 8 and Theorem 1, we can obtain the following result by using mathematical induction on $n$.

Theorem 2. Let $\tilde{\alpha}_{i}(i=1,2, \cdots, n)$ be a collection of triangular intuitionistic fuzzy number, then their aggregated value by using the TIFWG operator is also an triangular intuitionistic fuzzy number, and

$$
\begin{aligned}
& \operatorname{TIFWG}_{w}\left(\tilde{\alpha}_{1}, \tilde{\alpha}_{2}, \cdots, \tilde{\alpha}_{n}\right)= \\
& \left(\left[\prod_{i=1}^{n}\left(a_{i}\right)^{w_{i}}, \prod_{i=1}^{n}\left(b_{i}\right)^{w_{i}}, \prod_{i=1}^{n}\left(c_{i}\right)^{w_{i}}\right] ; \prod_{i=1}^{n}\left(u_{\tilde{\alpha}_{i}}\right)^{w_{i}}, 1-\prod_{i=1}^{n}\left(1-v_{\tilde{\alpha}_{i}}\right)^{w_{i}}\right)
\end{aligned}
$$

where $w=\left(w_{1}, w_{2}, \ldots, w_{n}\right)^{T}$ is the weight vector of $\tilde{\alpha}_{i}(i=1,2, \cdots, n)$, with $w_{i} \in[0,1]$ and $\sum_{i=1}^{n} w_{i}=1$

Proof. The first result follows quickly from Definition 8. In the following, we prove that Eq.(10) by using mathematical induction on $n$.

(1) For $n=2$ :

Since 


$$
\begin{aligned}
& \tilde{\alpha}_{1}^{\omega_{1}}=\left(\left[a_{1}^{\omega_{1}}, b_{1}^{\omega_{1}}, c_{1}^{\omega_{1}}\right] ;\left(u_{\tilde{\alpha}_{1}}\right)^{\omega_{1}}, 1-\left(1-v_{\tilde{\alpha}_{1}}\right)^{\omega_{1}}\right), \\
& \tilde{\alpha}_{2}^{\omega_{2}}=\left(\left[a_{2}^{\omega_{2}}, b_{2}^{\omega_{2}}, c_{2}^{\omega_{2}}\right] ;\left(u_{\tilde{\alpha}_{2}}\right)^{\omega_{2}}, 1-\left(1-v_{\tilde{\alpha}_{2}}\right)^{\omega_{2}}\right) .
\end{aligned}
$$

then

$$
\begin{aligned}
& \operatorname{TIFWG}\left(\tilde{\alpha}_{1}, \tilde{\alpha}_{2}\right)=\tilde{\alpha}_{1}^{\omega_{1}} \otimes \tilde{\alpha}_{2}^{\omega_{2}} \\
& =\left(\left[a_{1}^{\omega_{1}}, b_{1}^{\omega_{1}}, c_{1}^{\omega_{1}}\right] ;\left(u_{\tilde{\alpha}_{1}}\right)^{\omega_{1}}, 1-\left(1-v_{\tilde{\alpha}_{1}}\right)^{\omega_{1}}\right) \otimes\left(\left[a_{2}^{\omega_{2}}, b_{2}^{\omega_{2}}, c_{2}^{\omega_{2}}\right] ;\left(u_{\tilde{\alpha}_{2}}\right)^{\omega_{2}}, 1-\left(1-v_{\tilde{\alpha}_{2}}\right)^{\omega_{2}}\right) \\
& =\left(\begin{array}{l}
{\left[a_{1}^{\omega_{1}} a_{2}^{\omega_{2}}, b_{1}^{\omega_{1}} b_{2}^{\omega_{2}}, c_{1}^{\omega_{1}} c_{2}^{\omega_{2}}\right] ;\left(u_{\tilde{\alpha}_{1}}\right)^{\omega_{1}}\left(u_{\tilde{\alpha}_{2}}\right)^{\omega_{2}}, 1-\left(1-v_{\tilde{\alpha}_{1}}\right)^{\omega_{1}}+1-\left(1-v_{\tilde{\alpha}_{2}}\right)^{\omega_{2}}-} \\
\left(1-\left(1-v_{\tilde{\alpha}_{1}}\right)^{\omega_{1}}\right)\left(1-\left(1-v_{\tilde{\alpha}_{2}}\right)^{\omega_{2}}\right)
\end{array}\right) \\
& =\left(\left[a_{1}^{\omega_{1}} a_{2}^{\omega_{2}}, b_{1}^{\omega_{1}} b_{2}^{\omega_{2}}, c_{1}^{\omega_{1}} c_{2}^{\omega_{2}}\right] ;\left(u_{\tilde{\alpha}_{1}}\right)^{\omega_{1}}\left(u_{\tilde{\alpha}_{2}}\right)^{\omega_{2}}, 1-\left(1-v_{\tilde{\alpha}_{1}}\right)^{\omega_{1}}\left(1-v_{\tilde{\alpha}_{2}}\right)^{\omega_{2}}\right)
\end{aligned}
$$

Thus, Eq.(10) holds.

(2) If Eq.(10) holds for $n=k$, that is

$$
\begin{aligned}
& \operatorname{TIFWG}\left(\tilde{\alpha}_{1}, \tilde{\alpha}_{2}, \cdots, \tilde{\alpha}_{k}\right)=\prod_{i=1}^{k} \tilde{\alpha}_{i}^{\omega_{i}} \\
& \left.=\left(\left[\prod_{i=1}^{k}\left(a_{i}\right)^{\omega_{i}}, \prod_{i=1}^{k}\left(b_{i}\right)^{\omega_{i}}, \prod_{i=1}^{k}\left(c_{i}\right)^{\omega_{i}}\right)\right] ; \prod_{i=1}^{k}\left(u_{\tilde{\alpha}_{i}}\right)^{\omega_{i}}, 1-\prod_{i=1}^{k}\left(1-v_{\tilde{\alpha}_{i}}\right)^{\omega_{i}}\right)
\end{aligned}
$$

then, when $n=k+1$, by the operational laws in Definition 2, we have

$$
\begin{aligned}
& \operatorname{TIFWG}\left(\tilde{\alpha}_{1}, \tilde{\alpha}_{2}, \cdots, \tilde{\alpha}_{k+1}\right)=\prod_{i=1}^{k+1} \tilde{\alpha}_{i}^{\omega_{i}}=\operatorname{TIFWG}\left(\tilde{\alpha}_{1}, \tilde{\alpha}_{2}, \cdots, \tilde{\alpha}_{k}\right) \otimes \tilde{\alpha}_{k+1}{ }^{\omega_{k+1}} \\
& \left.=\left(\left[\prod_{i=1}^{k}\left(a_{i}\right)^{\omega_{i}}, \prod_{i=1}^{k}\left(b_{i}\right)^{\omega_{i}}, \prod_{i=1}^{k}\left(c_{i}\right)^{\omega_{i}}\right)\right] ; \prod_{i=1}^{k}\left(u_{\tilde{\alpha}_{i}}\right)^{\omega_{i}}, 1-\prod_{i=1}^{k}\left(1-v_{\tilde{\alpha}_{i}}\right)^{\omega_{i}}\right) \otimes \\
& =\left(\left[a_{k+1}^{\omega_{k+1}}, b_{k+1}^{\omega_{k+1}}, c_{k+1}^{\omega_{k+1}}\right] ;\left(u_{\tilde{\alpha}_{k+1}}\right)^{\omega_{k+1}},\left(v_{\tilde{\alpha}_{k+1}}\right)^{\omega_{k+1}}\right) \\
& \left.1-\prod_{i=1}^{k}\left(a_{i}\right)^{\omega_{i}} \otimes a_{k+1}{ }^{\omega_{k+1}}, \prod_{i=1}^{k}\left(b_{i}\right)^{\omega_{i}} \otimes b_{k+1}{ }^{\omega_{k+1}}, \prod_{i=1}^{k}\left(c_{i}\right)^{\omega_{i}} \otimes c_{k+1}{ }^{\omega_{k+1}}\right] ; \prod_{i=1}^{k}\left(u_{\tilde{\alpha}_{i}}\right)^{\omega_{i}} \otimes\left(1-\left(1-v_{\tilde{\alpha}_{k+1}}\right)^{\omega_{k+1}}-\left(1-\prod_{i=1}^{k}\left(1-v_{\tilde{\alpha}_{i}}\right)^{w_{k+1}}\right)\left(1-\left(1-v_{\tilde{\alpha}_{k+1}}\right)^{\omega_{k+1}}\right)\right) \\
& =\left(\left[\prod_{i=1}^{k+1}\left(a_{i}\right)^{\omega_{i}}, \prod_{i=1}^{k+1}\left(b_{i}\right)^{\omega_{i}}, \prod_{i=1}^{k+1}\left(c_{i}\right)^{\omega_{i}}\right] ; \prod_{i=1}^{k+1}\left(u_{\tilde{\alpha}_{i}}\right)^{\omega_{i}}, 1-\prod_{i=1}^{k+1}\left(1-v_{\tilde{\alpha}_{i}}\right)^{\omega_{i}}\right)
\end{aligned}
$$

i.e. Eq.(10) holds for $n=k+1$.Thus, Eq.(10) holds for all $n$, which completes the proof of Theorem 2.

To study some desirable properties of TIFWG operator, we have the following theorem.

Theorem 3 (Idempotency). Let $\tilde{\alpha}_{i}(i=1,2, \cdots, n)$ be a collection of triangular intuitionistic fuzzy numbers, and $\omega=\left(\omega_{1}, \omega_{2}, \ldots, \omega_{n}\right)^{T}$ is the weight vector of $\tilde{\alpha}_{i}(i=1,2, \cdots, n)$, with $\sum_{i=1}^{n} \omega_{i}=1, \omega_{i} \in[0,1]$. If all $\tilde{\alpha}_{i}(i=1,2, \cdots, n)$ are equal, i.e. $\tilde{\alpha}_{i}=\tilde{\alpha}$ for all $i$, then

Proof. By Theorem 1, we have

$$
\operatorname{TIFWG}\left(\tilde{\alpha}_{1}, \tilde{\alpha}_{2}, \cdots, \tilde{\alpha}_{n}\right)=\tilde{\alpha}
$$


$\operatorname{TIFWG}\left(\tilde{\alpha}_{1}, \tilde{\alpha}_{2}, \cdots, \tilde{\alpha}_{n}\right)=\tilde{\alpha}_{1}^{\omega_{1}} \otimes \tilde{\alpha}_{2}^{\omega_{2}} \otimes \cdots \otimes \tilde{\alpha}_{n}^{\omega_{n}}=\tilde{\alpha}^{\sum_{i=1}^{n} \omega_{i}}=\tilde{\alpha}$.

Theorem 4 (Boundary). Let $\tilde{\alpha}_{i}(i=1,2, \cdots, n)$ be a collection of triangular intuitionistic fuzzy numbers, and let

$$
\begin{aligned}
& \tilde{\alpha}^{-}=\left(\left[\min _{i} a_{i}, \min _{i} b_{i}, \min _{i} c_{i}\right], \min u_{i}, \max v_{i}\right), \\
& \tilde{\alpha}^{+}=\left(\left[\max _{i} a_{i}, \max _{i} b_{i}, \max _{i} c_{i}\right], \max u_{i}, \min v_{i}\right) .
\end{aligned}
$$

then

$$
\tilde{\alpha}^{-} \leq \operatorname{TIFWG}\left(\tilde{\alpha}_{1}, \tilde{\alpha}_{2}, \cdots, \tilde{\alpha}_{n}\right) \leq \tilde{\alpha}^{+} .
$$

Proof. Since $\min _{i} a_{i} \leq a_{i} \leq \max _{i} a_{i}, \min _{i} b_{i} \leq b_{i} \leq \max _{i} b_{i}, \min _{i} c_{i} \leq c_{i} \leq \max _{i} c_{i}$, $\min _{i} \mu_{i} \leq \mu_{i} \leq \max _{i} \mu_{i}, \min _{i} v_{i} \leq v_{i} \leq \max _{i} v_{i}$ for all $i$, then

$$
\prod_{i=1}^{n}\left(a_{i}\right)^{\omega_{i}} \leq \prod_{i=1}^{n}\left(\max _{i} a_{i}\right)^{\omega_{i}}=\max _{i} a_{i}, \prod_{i=1}^{n}\left(a_{i}\right)^{\omega_{i}} \geq \prod_{i=1}^{n}\left(\min _{i} a_{i}\right)^{\omega_{i}}=\min _{i} a_{i}
$$

that is,

$$
\min _{i} a_{i} \leq \prod_{i=1}^{n}\left(a_{i}\right)^{\omega_{i}} \leq \max _{i} a_{i}
$$

Similarly, we have

$$
\begin{gathered}
\min _{i} b_{i} \leq \prod_{i=1}^{n}\left(b_{i}\right)^{\omega_{i}} \leq \max _{i} b_{i}, \quad \min _{i} c_{i} \leq \prod_{i=1}^{n}\left(c_{i}\right)^{\omega_{i}} \leq \max _{i} c_{i}, \\
\min _{i} u_{i} \leq \prod_{i=1}^{n}\left(u_{i}\right)^{\omega_{i}} \leq \max _{i} u_{i}, \quad \min _{i} v_{i} \leq 1-\prod_{i=1}^{n}\left(1-v_{i}\right)^{\omega_{i}} \leq \max _{i} v_{i} .
\end{gathered}
$$

Let $\operatorname{TIFWG}\left(\tilde{\alpha}_{1}, \tilde{\alpha}_{2}, \cdots, \tilde{\alpha}_{n}\right)=\left([a, b, c] ; u_{\tilde{\alpha}}, v_{\tilde{\alpha}}\right)$, then

$$
\begin{aligned}
& S(\tilde{\alpha})=E(\tilde{\alpha}) *\left(\mu_{\tilde{\alpha}}-v_{\tilde{\alpha}}\right)=\frac{a+2 b+c}{4}\left(1+\mu_{\tilde{\alpha}}-v_{\tilde{\alpha}}\right)\left(\mu_{\tilde{\alpha}}-v_{\tilde{\alpha}}\right) \\
& \leq \frac{\max _{i} a_{i}+\max _{i} 2 b_{i}+\max _{i} c_{i}}{4}\left(1+\max _{i} \mu_{\tilde{\alpha}_{i}}-\min _{i} v_{\tilde{\alpha}_{i}}\right)\left(\max _{i} \mu_{\tilde{\alpha}_{i}}-\min _{i} v_{\tilde{\alpha}_{i}}\right. \\
& =S\left(\tilde{\alpha}^{+}\right) \\
& S(\tilde{\alpha})=E(\tilde{\alpha}) *\left(\mu_{\tilde{\alpha}}-v_{\tilde{\alpha}}\right)=\frac{a+2 b+c}{4}\left(1+\mu_{\tilde{\alpha}}-v_{\tilde{\alpha}}\right)\left(\mu_{\tilde{\alpha}}-v_{\tilde{\alpha}}\right) \\
& \geq \frac{\min _{i} a_{i}+\min _{i} 2 b_{i}+\min _{i} c_{i}}{4}\left(1+\min _{i} \mu_{\tilde{\alpha}_{i}}-\max _{i} v_{\tilde{\alpha}_{i}}\right)\left(\min _{i} \mu_{\tilde{\alpha}_{i}}-\max _{i} v_{\tilde{\alpha}_{i}}\right) \\
& =S\left(\tilde{\alpha}^{-}\right)
\end{aligned}
$$

If $S(\tilde{\alpha})<S\left(\tilde{\alpha}^{+}\right)$and $S(\tilde{\alpha})>S\left(\tilde{\alpha}^{-}\right)$, then we have

$$
\tilde{\alpha}^{-}<\operatorname{TIFWG}_{w}\left(\tilde{\alpha}_{1}, \tilde{\alpha}_{2}, \cdots, \tilde{\alpha}_{n}\right)<\tilde{\alpha}^{+} .
$$

If $S_{\theta}(\tilde{\alpha})=S_{\theta}\left(\tilde{\alpha}^{+}\right)$,i.e., 


$$
\begin{aligned}
& \frac{a+2 b+c}{4}\left(1+\mu_{\tilde{\alpha}}-v_{\tilde{\alpha}}\right)\left(\mu_{\tilde{\alpha}}-v_{\tilde{\alpha}}\right) \\
& =\frac{\max _{i} a_{i}+\max _{i} 2 b_{i}+\max _{i} c_{i}}{4}\left(1+\max _{i} \mu_{\tilde{\alpha}_{i}}-\min _{i} v_{\tilde{\alpha}_{i}}\right)\left(\max _{i} \mu_{\tilde{\alpha}_{i}}-\min _{i} v_{\tilde{\alpha}_{i}}\right)
\end{aligned}
$$

then, we have

$$
a=\max _{i} a_{i}, b=\max _{i} b_{i}, c=\max _{i} c_{i}, \mu_{i}=\max _{i} \mu_{i}, v_{i}=\min _{i} v_{i}
$$

Therefore,

$$
\begin{aligned}
& H(\tilde{\alpha})=E(\tilde{\alpha}) *\left(\mu_{\tilde{\alpha}}+v_{\tilde{\alpha}}\right)=\frac{a+2 b+c}{4}\left(1+\mu_{\tilde{\alpha}}-v_{\tilde{\alpha}}\right)\left(\mu_{\tilde{\alpha}}+v_{\tilde{\alpha}}\right) \\
& =\frac{\max _{i} a_{i}+\max _{i} 2 b_{i}+\max _{i} c_{i}}{4}\left(1+\max _{i} \mu_{\tilde{\alpha}_{i}}-\min _{i} v_{\tilde{\alpha}_{i}}\right)\left(\max _{i} \mu_{\tilde{\alpha}_{i}}+\min _{i} v_{\tilde{\alpha}_{i}}\right) \\
& =H\left(\tilde{\alpha}^{+}\right)
\end{aligned}
$$

Similarly, we have

$$
\begin{aligned}
& H(\tilde{\alpha})=E(\tilde{\alpha}) *\left(\mu_{\tilde{\alpha}}+v_{\tilde{\alpha}}\right)=\frac{a+2 b+c}{4}\left(1+\mu_{\tilde{\alpha}}-v_{\tilde{\alpha}}\right)\left(\mu_{\tilde{\alpha}}+v_{\tilde{\alpha}}\right) \\
& =\frac{\min _{i} a_{i}+\max _{i} 2 b_{i}+\max _{i} c_{i}}{4}\left(1+\min _{i} \mu_{\tilde{\alpha}_{i}}-\max _{i} v_{\tilde{\alpha}_{i}}\right)\left(\min _{i} \mu_{\tilde{\alpha}_{i}}+\max _{i} v_{\tilde{\alpha}_{i}}\right) \\
& =H\left(\tilde{\alpha}^{-}\right)
\end{aligned}
$$

Then we have

$$
\operatorname{TIFWG}\left(\tilde{\alpha}_{1}, \tilde{\alpha}_{2}, \cdots, \tilde{\alpha}_{n}\right)=\tilde{\alpha}^{+} .
$$

Similarity, it follows that

$$
\operatorname{TIFWG}\left(\tilde{\alpha}_{1}, \tilde{\alpha}_{2}, \cdots, \tilde{\alpha}_{n}\right)=\tilde{\alpha}^{-} .
$$

Thus, we know that Eq.(12) always holds.

Theorem 5. (Monotonicity). Let $\tilde{\alpha}_{i}(i=1,2, \cdots, n)$ and $\tilde{\alpha}_{i}^{*}(i=1,2, \cdots, n)$ be a collection of triangular intuitionistic fuzzy numbers. If $\tilde{\alpha}_{i} \leq \tilde{\alpha}_{i}^{*}$ for all $i$, then

$$
\operatorname{TIFWG}\left(\tilde{\alpha}_{1}, \tilde{\alpha}_{2}, \cdots, \tilde{\alpha}_{n}\right) \leq \operatorname{TIFWG}\left(\tilde{\alpha}_{1}^{*}, \tilde{\alpha}_{2}^{*}, \cdots, \tilde{\alpha}_{n}^{*}\right)
$$

Based on Definition 2 and the ordered weighted geometric averaging (OWG) operator [23], we shall develop and triangular intuitionistic fuzzy version of the OWG operator.

Definition 9. Let $\tilde{\alpha}_{i}(i=1,2, \cdots, n)$ be a collection of triangular intuitionistic fuzzy numbers. A triangular intuitionistic fuzzy ordered weighted geometric (TIFOWG) operator of dimension $n$ is a mapping TIFOWG: $\Omega^{n} \rightarrow \Omega$, if

$$
\operatorname{TIFOWG}\left(\tilde{\alpha}_{1}, \tilde{\alpha}_{2}, \cdots, \tilde{\alpha}_{n}\right)=\prod_{i=1}^{n} \tilde{\alpha}_{\sigma(i)}^{w_{i}}
$$

where $(\sigma(1), \sigma(2), \cdots \sigma(n))$ is a permutation of $(1,2, \cdots, n)$ such that $\tilde{\alpha}_{\sigma(i-1)} \geq \tilde{\alpha}_{\sigma(i)}$ for all $i$, and $w=\left(w_{1}, w_{2}, \ldots, w_{n}\right)^{T}$ is the weight vector of $\tilde{\alpha}_{i}(i=1,2, \cdots, n)$, $\sum_{i=1}^{n} w_{i}=1, w_{i} \in[0,1]$. Especially, if $w=(1 / n, 1 / n, \ldots, 1 / n)^{T}$, the TIFOWG operator is reduced to the geometric averaging operator of the triangular intuitionistic fuzzy number 
(TIFGA).

Similar to Theorem 2, we have the following result.

Theorem 6. Let $\tilde{\alpha}_{i}(i=1,2, \cdots, n)$ be a collection of triangular intuitionistic fuzzy numbers, then their aggregated value by using the TIFOWG operator is also an triangular intuitionistic fuzzy number, and

$$
\begin{aligned}
& \text { TIFOWG }\left(\tilde{\alpha}_{1}, \tilde{\alpha}_{2}, \cdots, \tilde{\alpha}_{n}\right) \\
& =\left(\left[\prod_{i=1}^{n}\left(a_{\sigma(i)}\right)^{w_{i}}, \prod_{i=1}^{n}\left(b_{\sigma(i)}\right)^{w_{i}}, \prod_{i=1}^{n}\left(c_{\sigma(i)}\right)^{w_{i}}\right] ; \prod_{i=1}^{n}\left(u_{\tilde{\alpha}_{\sigma(i)}}\right)^{w_{i}}, 1-\prod_{i=1}^{n}\left(1-v_{\tilde{\alpha}_{\sigma(i)}}\right)^{w_{i}}\right)
\end{aligned}
$$

where $w=\left(w_{1}, w_{2}, \ldots, w_{n}\right)^{T}$ is the weighting vector of TIFOWG operator, with $\sum_{i=1}^{n} w_{i}=1$ and $w_{i} \in[0,1]$.

The TIFOWG operator has the following properties, which is similar to those of the TIFWG operator.

Theorem 7 (Idempotency). Let $\tilde{\alpha}_{i}(i=1,2, \cdots, n)$ be a collection of triangular intuitionistic fuzzy numbers, and $w=\left(w_{1}, w_{2}, \ldots, w_{n}\right)^{T}$ is the weight vector of TIFOWG operator, $\sum_{i=1}^{n} w_{i}=1, w_{i} \in[0,1]$. If all $\tilde{\alpha}_{i}(i=1,2, \cdots, n)$ are equal, i.e. $\tilde{\alpha}_{i}=\tilde{\alpha}$ for all $i$, then

$$
\mathrm{TIFOWG}_{w}\left(\tilde{\alpha}_{1}, \tilde{\alpha}_{2}, \cdots, \tilde{\alpha}_{n}\right)=\tilde{\alpha}
$$

Theorem 8 (Boundary). Let $\tilde{\alpha}_{i}(i=1,2, \cdots, n)$ be a collection of triangular intuitionistic fuzzy numbers, and let

$$
\begin{aligned}
& \tilde{\alpha}^{-}=\left(\left[\min _{i} a_{i}, \min _{i} b_{i}, \min _{i} c_{i}\right], \min u_{i}, \max v_{i}\right), \\
& \tilde{\alpha}^{+}=\left(\left[\max _{i} a_{i}, \max _{i} b_{i}, \max _{i} c_{i}\right], \max u_{i}, \min v_{i}\right) .
\end{aligned}
$$

then

$$
\tilde{\alpha}^{-} \leq \mathrm{TIFOWG}_{w}\left(\tilde{\alpha}_{1}, \tilde{\alpha}_{2}, \cdots, \tilde{\alpha}_{n}\right) \leq \tilde{\alpha}^{+}
$$

Theorem 9. (Monotonicity). Let $\tilde{\alpha}_{i}(i=1,2, \cdots, n)$ and $\tilde{\alpha}_{i}^{*}(i=1,2, \cdots, n)$ be a collection of triangular intuitionistic fuzzy numbers. If $\tilde{\alpha}_{i} \leq \tilde{\alpha}_{i}^{*}$ for all $i$, then

$$
\operatorname{TIFOWG}\left(\tilde{\alpha}_{1}, \tilde{\alpha}_{2}, \cdots, \tilde{\alpha}_{n}\right) \leq \operatorname{TIFOWG}\left(\tilde{\alpha}_{1}^{*}, \tilde{\alpha}_{2}^{*}, \cdots, \tilde{\alpha}_{n}^{*}\right)
$$

Theorem 10. (Commutativity). Let $\tilde{\alpha}_{i}(i=1,2, \cdots, n)$ and $\tilde{\alpha}_{i}^{*}(i=1,2, \cdots, n)$ be a collection of triangular intuitionistic fuzzy numbers, then

$$
\operatorname{TIFOWG}\left(\tilde{\alpha}_{1}, \tilde{\alpha}_{2}, \cdots, \tilde{\alpha}_{n}\right)=\operatorname{TIFOWG}\left(\tilde{\alpha}_{1}^{*}, \tilde{\alpha}_{2}^{*}, \cdots, \tilde{\alpha}_{n}^{*}\right)
$$

where $\quad \tilde{\alpha}_{i}^{*}(i=1,2, \cdots, n) \quad$ is any permutation of $\quad \tilde{\alpha}_{i}(i=1,2, \cdots, n)$,and $w=\left(w_{1}, w_{2}, \ldots, w_{n}\right)^{T}$ is the weight vector of TIFOWG, with $\sum_{i=1}^{n} w_{i}=1, w_{i} \in[0,1]$.

Proof. Let

$$
\operatorname{TIFOWG}\left(\tilde{\alpha}_{1}, \tilde{\alpha}_{2}, \cdots, \tilde{\alpha}_{n}\right)=\prod_{i=1}^{n} \tilde{\alpha}_{\sigma(i)}^{w_{i}}
$$




$$
\mathrm{TIFOWG}_{w}\left(\tilde{\alpha}_{1}^{*}, \tilde{\alpha}_{2}^{*}, \cdots, \tilde{\alpha}_{n}^{*}\right)=\prod_{i=1}^{n} \tilde{\alpha}_{\sigma(i)}^{* w_{i}}
$$

since $\tilde{\alpha}_{i}^{*}(i=1,2, \cdots, n)$ is any permutation of $\tilde{\alpha}_{i}(i=1,2, \cdots, n)$, we have

$$
\tilde{\alpha}_{\sigma(i)}^{*}=\tilde{\alpha}_{\sigma(i)}(i=1,2, \cdots, n)
$$

then Eq.(21) holds..

By Definitions 8 and 9, we know that the TIFWG operator weights only the triangular intuitionistic fuzzy numbers, while the TIFOWG operator weights only the ordered positions of the triangular intuitionistic fuzzy numbers. To overcome this limitation, we shall develop a triangular intuitionistic fuzzy hybrid geometric (TIFHG) operator.

Definition 10. Let $\tilde{\alpha}_{i}(i=1,2, \cdots, n)$ be a collection of triangular intuitionistic fuzzy numbers. A triangular intuitionistic fuzzy hybrid geometric (TIFHG) operator of dimension $n$ is a mapping TIFHG: $\Omega^{n} \rightarrow \Omega$, which has an associated vector $w=\left(w_{1}, w_{2}, \ldots, w_{n}\right)^{T}$ with $\sum_{i=1}^{n} w_{i}=1, w_{i} \in[0,1]$ such that

$$
\operatorname{ITFHG}_{\omega, w}\left(\tilde{\alpha}_{1}, \tilde{\alpha}_{2}, \cdots, \tilde{\alpha}_{n}\right)=\tilde{\beta}_{\sigma(1)}{ }^{w_{1}} \otimes \tilde{\beta}_{\sigma(2)}{ }^{w_{2}} \otimes \cdots \otimes \tilde{\beta}_{\sigma(n)}{ }^{w_{n}}
$$

where $\tilde{\beta}_{\sigma(i)}$ is the $i$ th largest of the weighted triangular intuitionistic fuzzy number $\tilde{\beta}_{i}\left(\tilde{\beta}_{i}=\tilde{\alpha}_{i}^{n \omega_{i}}, i=1,2, \cdots, n\right), \omega=\left(\omega_{1}, \omega_{2}, \cdots, \omega_{n}\right)^{T}$ is weight vector of $\tilde{\alpha}_{i}(i=1,2, \cdots, n)$ with $\sum_{i=1}^{n} w_{i}=1, \omega_{i} \in[0,1]$, and $n$ is the balancing coefficient, which plays a role of balance.

Similar to Theorem 2, we also obtain the following result.

Theorem 11. Let $\tilde{\alpha}_{i}(i=1,2, \cdots, n)$ be a collection of triangular intuitionistic fuzzy numbers, then, we have

$$
\begin{aligned}
& \text { ITFHG }_{\omega, w}\left(\tilde{\alpha}_{1}, \tilde{\alpha}_{2}, \cdots, \tilde{\alpha}_{n}\right) \\
& =\left(\left[\prod_{i=1}^{n} a_{\sigma(i)}^{\prime}{ }^{w_{i}}, \prod_{i=1}^{n} b_{\sigma(i)}^{\prime}{ }^{w_{i}}, \prod_{i=1}^{n} c_{\sigma(i)}^{\prime}{ }^{w_{i}}\right] ; \prod_{i=1}^{n}\left(u_{\tilde{\beta}_{\sigma(i)}}\right)^{w_{i}}, 1-\prod_{i=1}^{n}\left(1-v_{\tilde{\beta}_{\sigma(i)}}\right)^{w_{i}}\right)
\end{aligned}
$$

and the aggregated value derived by using the TIFHG operator is also an triangular intuitionistic fuzzy number.

Theorem 12. The TIFWG operator is a special case of the TIFHG operator.

Proof. Let $\omega=(1 / n, 1 / n, \ldots, 1 / n)^{T}$, then

$$
\begin{aligned}
& \operatorname{ITFHG}\left(\tilde{\alpha}_{1}, \tilde{\alpha}_{2}, \cdots, \tilde{\alpha}_{n}\right)=\tilde{\beta}_{\sigma(1)}^{w_{1}} \otimes \tilde{\beta}_{\sigma(2)}{ }^{w_{2}} \otimes \cdots \otimes \tilde{\beta}_{\sigma(n)}{ }^{w_{n}} \\
& =\left(\tilde{\beta}_{\sigma(1)} \otimes \tilde{\beta}_{\sigma(2)} \otimes \cdots \otimes \tilde{\beta}_{\sigma(n)}\right)^{1 / n} \\
& =\tilde{\alpha}_{1}^{\omega_{1}} \otimes \tilde{\alpha}_{2}^{\omega_{2}} \otimes \cdots \otimes \tilde{\alpha}_{n}^{\omega_{n}}=\operatorname{ITFWG}\left(\tilde{\alpha}_{1}, \tilde{\alpha}_{2}, \cdots, \tilde{\alpha}_{n}\right)
\end{aligned}
$$

Theorem 13. The TIFOWG operator is a special case of the TIFHG operator.

Proof. Let $w=(1 / n, 1 / n, \ldots, 1 / n)^{T}$, then $\tilde{\beta}_{i}=\tilde{\alpha}_{i}, i=1,2, \cdots, n$, thus 


$$
\begin{aligned}
& \operatorname{ITFHG}\left(\tilde{\alpha}_{1}, \tilde{\alpha}_{2}, \cdots, \tilde{\alpha}_{n}\right)=\tilde{\beta}_{\sigma(1)}^{w_{1}} \otimes \tilde{\beta}_{\sigma(2)}^{w_{2}} \otimes \cdots \otimes \tilde{\beta}_{\sigma(n)}^{w_{n}} \\
& =\tilde{\alpha}_{\sigma(1)}^{w_{1}} \otimes \tilde{\alpha}_{\sigma(2)}^{w_{2}} \otimes \cdots \otimes \tilde{\alpha}_{\sigma(n)}^{w_{n}} \\
& =\operatorname{ITFOWG}\left(\tilde{\alpha}_{1}, \tilde{\alpha}_{2}, \cdots, \tilde{\alpha}_{n}\right)
\end{aligned}
$$

\section{Multi-criteria decision making method based on triangular intuitionistic fuzzy numbers}

In this section, based on the TIFWG, TIFOWG and TIFHG operators, we will propose a new method to solve multi-criteria decision making problems in which the ratings of alternatives on criteria are expressed using TIFNs.

For some fuzzy multi-criteria decision making problem, let $A=\left\{A_{1}, A_{2}, \ldots, A_{m}\right\}$ be a discrete set of alternatives, and $C=\left\{C_{1}, C_{2}, \ldots, C_{n}\right\}$ be the set of criteria, $\omega=\left(\omega_{1}, \omega_{2}, \cdots, \omega_{n}\right)^{T}$ is the corresponding weight vector of the criteria, where $\omega_{j} \in[0,1], \sum_{j=1}^{n} \omega_{j}=1$, but the value $\omega_{j}$ is unknown. The criteria value of alternative $A_{i}$ on the criteria $C_{j}$ is the triangular intuitionistic fuzzy number $\tilde{h}_{i j}=\left(\left[a_{i j}, b_{i j}, c_{i j}\right] ; u_{i j}, v_{i j}\right), u_{i j}+v_{i j} \leq 1, v_{i j} \in[0,1], u_{i j} \in[0,1], \quad i=1,2, \cdots, m$, $j=1,2, \cdots, n$, and the decision matrix denotes $H=\left[\tilde{h}_{i j}\right]_{m \times n}$ is constructed. Assuming decision maker have indifferent risk preference.

\subsection{Standardize decision matrix}

To eliminate the effect from different physical dimensions to decision results, the decision making matrix $H=\left[\tilde{h}_{i j}\right]_{m \times n}$ should be standardized firstly. Suppose that the standardized decision matrix is $\tilde{R}=\left[\tilde{r}_{i j}\right]_{m \times n}, \quad \tilde{r}_{i j}=\left(\left[r_{i j}^{1}, r_{i j}^{2}, r_{i j}^{3}\right] ; u_{i j}, v_{i j}\right)$. In general, the criteria can be classified into two common types: benefit type and cost type, then the standardized methods are shown as follows:

(1) For benefit type of criteria:

$$
\tilde{r}_{i j}=\left(\left[\frac{h_{i j}^{1}-h_{j}^{-}}{h_{j}^{+}-h_{j}^{-}}, \frac{h_{i j}^{2}-h_{j}^{-}}{h_{j}^{+}-h_{j}^{-}}, \frac{h_{i j}^{3}-h_{j}^{-}}{h_{j}^{+}-h_{j}^{-}}\right] ; u_{i j}, v_{i j}\right)
$$

(2) For cost type of criteria:

$$
\tilde{r}_{i j}=\left(\left[\frac{h_{j}^{+}-h_{i j}^{3}}{h_{j}^{+}-h_{j}^{-}}, \frac{h_{j}^{+}-h_{i j}^{2}}{h_{j}^{+}-h_{j}^{-}}, \frac{h_{j}^{+}-h_{i j}^{1}}{h_{j}^{+}-h_{j}^{-}}\right] ; u_{i j}, v_{i j}\right)
$$

where $h_{j}^{+}=\max _{i}\left(h_{i j}^{3}\right), h_{j}^{-}=\min _{i}\left(h_{i j}^{1}\right), i=1,2, \cdots, m, j=1,2, \cdots, n$ 。 


\subsection{Determine the criteria weight}

There are many methods to determine the criteria weight, such as maximizing deviation method [31], information entropy method[19] and other optimization method[30]. In this paper, we will adopt maximizing deviation method to determine the criteria weight.

The maximizing deviation method is proposed by Wang[31] to deal with multi-criteria decision making problems with numerical information. For a multi-criteria decision making problem, we need to compare the collective preference values to rank the alternatives, the larger the ranking value $\tilde{r}_{i}$ is, the better the corresponding alternative $A_{i}$ is. If the criteria values of all alternative have little differences under a criteria, it shows that such a criteria plays a small important role in the priority procedure. Contrariwise, if some criteria makes the performance values among all the alternatives have obvious differences, such an attribute plays an important role in choosing the best alternative. So to the view of sorting the alternatives, if one criteria has similar attribute values across alternatives, it should be assigned a small weight; otherwise, the criteria which makes larger deviations should be evaluated a bigger weight, in spite of the degree of its own importance. Especially, if all available alternatives score about equally with respect to a given attribute, then such a criteria will be judged unimportant by most decision makers. In other word, such a criteria should be assigned a very small weight[35].

Because the traditional maximizing deviation method is generally suitable for criteria value taking the form of crisp number and yet it fail in dealing with the triangular intuitionistic fuzzy number. Therefore, the deviation method is selected here to compute the differences of the performance values of each alternative based on Definition 3. The steps of determining the attribute weights by the maximizing deviation method are shown as follows:

(1)For the criteria $C_{j}$, the deviation value $D_{j}(\omega)$ of all alternative to all the other alternatives can be defined as follows:

$$
D_{j}(\omega)=\sum_{i=1}^{m} D_{i j}(\omega)=\sum_{i=1}^{m} \sum_{l=1}^{m}\left|\tilde{r}_{i j}-\tilde{r}_{l j}\right| \omega_{j}
$$

(2)We can construct a non-linear programming model as follows:

$$
(\mathrm{M} 1) \begin{cases}\max & D\left(\omega_{j}\right)=\sum_{j=1}^{n} \sum_{i=1}^{m} \sum_{l=1}^{m}\left|\left(\tilde{r}_{i j}-\tilde{r}_{l j}\right)\right| \omega_{j} \\ \text { s.t } & \sum_{j=1}^{n}\left(\omega_{j}\right)^{2}=1, \omega_{j} \geq 0, j=1,2 \cdots, n\end{cases}
$$

(3)To solve the above model, let

$$
L\left(\omega_{j}, \zeta\right)=\sum_{j=1}^{n} \sum_{i=1}^{m} \sum_{l=1}^{m}\left|\tilde{r}_{i j}-\tilde{r}_{l j}\right| \omega_{j}+\frac{1}{2} \zeta\left(\sum_{j=1}^{n}\left(\omega_{j}\right)^{2}-1\right)
$$

denote the Lagrange function of the constrained optimization problem (M1), where $\xi$ is a real number. Then the partial derivatives of $L$ are computed as 


$$
\left\{\begin{array}{l}
\frac{\partial L}{\partial \omega_{j}}=\sum_{j=1}^{n} \sum_{i=1}^{m} \sum_{l=1}^{m}\left|\tilde{r}_{i j}-\tilde{r}_{l j}\right|++\zeta \omega_{j}=0 \\
\frac{\partial L}{\partial \zeta}=\frac{1}{2}\left(\sum_{j=1}^{n}\left(\omega_{j}\right)^{2}-1\right)=0
\end{array}\right.
$$

It can be verified easily that $\omega_{j}^{*}$ are positive such that they do satisfy the constrained conditions in model ( M1) and the solution is unique.

$$
\omega_{j}^{*}=\frac{\sum_{i=1}^{m} \sum_{l=1}^{m}\left|\tilde{r}_{i j}-\tilde{r}_{l j}\right|}{\sqrt{\sum_{j=1}^{n}\left[\sum_{i=1}^{m} \sum_{l=1}^{m}\left|\tilde{h}_{i j}-\tilde{h}_{l j}\right|\right]^{2}}} .
$$

By normalizing $\omega_{j}^{*}$ to let the sum of $\omega_{j}$, we have

$$
\omega_{j}=\frac{\sum_{i=1}^{m} \sum_{l=1}^{m}\left|h_{i j}-h_{l j}\right|}{\sum_{j=1}^{n} \sum_{i=1}^{m} \sum_{i=1}^{m}\left|h_{i j}-h_{l j}\right|} .
$$

Based on the above analysis, we develop a practical approach based on the TIFWG operator, the TIFOWG operator and the TIFHG operator to the multi-criteria decision making with triangular intuitionistic fuzzy information as follows:

(1) Construct the decision matrix $H=\left[\tilde{h}_{i j}\right]_{m \times n}$, where all the arguments $\tilde{h}_{i j}$ are triangular intuitionistic fuzzy numbers.

(2) To eliminate the effect from different physical dimensions by the Eq. (24)-Eq. (25), the decision matrix $H=\left[\tilde{h}_{i j}\right]_{m \times n}$ are converted to standardize decision matrix $\tilde{R}=\left[\tilde{r}_{i j}\right]_{m \times n}$

(3) If the weight vector of criteria is completely unknown, utilize Eq. (26) to obtain the optimal weight of criteria.

(4) Utilize the TIFWG operator, the TIFOWG operator and the TIFHG operator to aggregate the criteria values $\tilde{r}_{i j}(j=1,2, \cdots n)$ of the alternative $A_{i}$.

(5) Rank all the alternatives $A_{i} \quad(i=1,2, \cdots m)$ and select the best one(s) in accordance with score function values $S\left(\tilde{r}_{i}\right)$ and accuracy function values $H\left(\tilde{r}_{i}\right)$. The largest $S\left(\tilde{r}_{i}\right)$ and $H\left(\tilde{r}_{i}\right)$, the better the alternatives $A_{i}(i=1,2, \cdots m)$ will be.

(6) End.

\section{Numerical example}

A car company is desirable to select the most appropriate green supplier for one of the key elements in its manufacturing process. After pre-evaluation, four suppliers $A_{i}(i=1,2,3,4)$ have remained as alternatives for further evaluation. Three criteria are considered as: 
product quality $C_{1}$; technology capability $C_{2}$; environment management $C_{3}$; (whose weighting vector is completely unknown). They constructed the decision matrices $H=\left[\tilde{h}_{i j}\right]_{m \times n}$ as follows:

$$
H=\left[\begin{array}{lll}
([2,3,4] ; 0.7,0.2) & ([3,4,5] ; 0.5,0.4) & ([4,6,7] ; 0.7,0.2) \\
([5,6,7] ; 0.6,0.3) & ([3,5,6] ; 0.6,0.3) & ([6,7,9] ; 0.5,0.4) \\
([4,5,8] ; 0.5,0.4) & ([3,4,5] ; 0.8,0.2) & ([3,6,7] ; 0.6,0.4) \\
([3,5,7] ; 0.5,0.3) & ([4,5,6] ; 0.7,0.2) & ([5,6,8] ; 0.6,0.3)
\end{array}\right]
$$

Steps using the method in this article are as follows:

(1). Standardize triangular intuitionistic fuzzy decision matrix $\tilde{R}=\left[\tilde{r}_{i j}\right]_{m \times n}$ produced by Eq. (24)-Eq. (25) is shown as follows:

$$
R=\left[\begin{array}{ccc}
([0,0.17,0.33] ; 0.7,0.2) & ([0,0.33,0.67] ; 0.5,0.4) & ([0.17,0.5,0.67] ; 0.7,0.2) \\
([0.5,0.67,0.83] ; 0.6,0.3) & ([0,0.67,1] ; 0.6,0.3) & ([0.5,0.67,1] ; 0.5,0.4) \\
([0.33,0.5,1] ; 0.5,0.4) & ([0,0.33,0.67] ; 0.8,0.2) & ([0,0.5,0.67] ; 0.6,0.4) \\
([0.17,0.5,0.83] ; 0.5,0.3) & ([0.33,0.67,1] ; 0.7,0.2) & ([0.33,0.5,0.83] ; 0.6,0.3)
\end{array}\right]
$$

(2). Calculate the attribute weight $\omega$ by model (M1) is shown as follows:

$$
w=(0.381,0.405,0.214)
$$

(3). Applying the TIFWG operator, the TIFOWG operator and the TIFHG operator to derive the collective overall preference values $\tilde{r}_{i}$ of alternative $A_{i}$ (let $\left.w=(0.3,0.3,0.4)^{T}\right)$, as shown in Table 1 .

Table 1. The value of alternative $A_{i}$ by the different aggregation operator

\begin{tabular}{|c|c|c|c|}
\hline$A_{i}$ & TIFWG & TIFOWG & TIFHG \\
\hline$A_{1}$ & $([0,0.28,0.51] ; 0.61,0.29)$ & $([0,0.34,0.58] ; 0.61,0.29)$ & $([0,0.35,0.58] ; 0.62,0.28)$ \\
\hline$A_{2}$ & $([0,0.67,0.93] ; 0.58,0.32)$ & $([0,0.67,0.93] ; 0.58,0.32)$ & $([0,0.68,0.94] ; 0.58,0.32)$ \\
\hline$A_{3}$ & $([0,0.42,0.78] ; 0.63,0.33)$ & $([0,0.43,0.79] ; 0.62,0.33)$ & $([0,0.44,0.79] ; 0.63,0.33)$ \\
\hline$A_{4}$ & $([0.26,0.56,0.9] ; 0.6,0.26)$ & $([0.29,0.56,0.89] ; 0.61,0.26)$ & $([0.27,0.57,0.89] ; 0.6,0.26)$ \\
\hline
\end{tabular}

(3). Rank all the alternatives $A_{i}(i=1,2,3,4)$ by the score function values $S\left(\tilde{r}_{i}\right)$ and the accuracy function values $H\left(\tilde{r}_{i}\right)$ of triangular intuitionistic fuzzy values under the TIFWG operator, the TIFOWG operator, and the TIFHG operator, as shown in Table 2.

Then, we can derive the ranking of alternative by the score function values $S\left(\tilde{r}_{i}\right): A_{4} \succ A_{2} \succ A_{3} \succ A_{1}$, and thus the most desirable alternative is $A_{4}$.

In addition, in order to verify the validity of the method proposed in this paper, we use the criteria weight $\omega=(0.381,0.405,0.214)$ which are generated in this paper and use the proposed method[34], we can get the distances $d\left(\tilde{r}_{i}, \tilde{r}^{+}\right)$between collective overall 
values $\tilde{r}_{i}$ and the positive ideal solution, as shown in Table 3 .

From the Table 3, we can derive the ranking of alternatives: $A_{4} \succ A_{2} \succ A_{3} \succ A_{1}$.

However, when we use the triangular intuitionistic fuzzy weighted averaging (TIFWA) operator, the triangular intuitionistic fuzzy ordered weighted averaging (TIFOWA) operator, he triangular intuitionistic fuzzy hybrid aggregation (TIFHA) operator[21] and the ranking method which are generated in this paper, we can get the collective overall values $\tilde{r}_{i}$ and score function values $S\left(\tilde{r}_{i}\right)$, as seen in shown 4 .

Table 2. The ranking results of alternatives by the score function

\begin{tabular}{|c|c|c|c|}
\hline$A_{i}$ & TIFWG & TIFOWG & TIFHG \\
\hline$S\left(\tilde{r}_{1}\right)$ & 0.056 & 0.066 & 0.072 \\
\hline$S\left(\tilde{r}_{2}\right)$ & 0.092 & 0.090 & 0.094 \\
\hline$S\left(\tilde{r}_{3}\right)$ & 0.079 & 0.077 & 0.082 \\
\hline$S\left(\tilde{r}_{4}\right)$ & 0.129 & 0.135 & 0.131 \\
\hline & $A_{4} \succ A_{2} \succ A_{3} \succ A_{1}$ & $A_{4} \succ A_{2} \succ A_{3} \succ A_{1}$ & $A_{4} \succ A_{2} \succ A_{3} \succ A_{1}$ \\
\hline
\end{tabular}

Table 3. The ranking results of alternatives by the distances $d\left(\tilde{r}_{i}, \tilde{r}^{+}\right)$

\begin{tabular}{|c|c|c|c|}
\hline$A_{i}$ & TIFWG & TIFOWG & TIFHG \\
\hline$d\left(\tilde{r}_{1}, \tilde{r}^{+}\right)$ & 0.826 & 0.798 & 0.792 \\
\hline$d\left(\tilde{r}_{2}, \tilde{r}^{+}\right)$ & 0.664 & 0.664 & 0.660 \\
\hline$d\left(\tilde{r}_{3}, \tilde{r}^{+}\right)$ & 0.740 & 0.738 & 0.734 \\
\hline$d\left(\tilde{r}_{4}, \tilde{r}^{+}\right)$ & 0.616 & 0.609 & 0.614 \\
\hline & $A_{4} \succ A_{2} \succ A_{3} \succ A_{1}$ & $A_{4} \succ A_{2} \succ A_{3} \succ A_{1}$ & $A_{4} \succ A_{2} \succ A_{3} \succ A_{1}$ \\
\hline
\end{tabular}

Table 4. The value of alternative $A_{i}$ by the different aggregation operator[21]

\begin{tabular}{|c|c|c|c|}
\hline$A_{i}$ & TIFWA & TIFOWA & TIFHA \\
\hline$A_{1}$ & $([0.04,0.31,0.54] ; 0.63,0.26)$ & $([0.06,0.36,0.6] ; 0.63,0.26)$ & $([0.08,0.38,0.61] ; 0.64,0.25)$ \\
\hline$A_{2}$ & $([0.3,0.67,0.94] ; 0.58,0.32)$ & $([0.3,0.67,0.94] ; 0.58,0.32)$ & $([0.41,0.68,0.95] ; 0.56,0.34)$ \\
\hline$A_{3}$ & $([0.13,0.43,0.8] ; 0.58,0.32)$ & $([0.13,0.44,0.8] ; 0.66,0.31)$ & $([0.06,0.45,0.74] ; 0.68,0.31)$ \\
\hline$A_{4}$ & $([0.27,0.57,0.9] ; 0.61,0.25)$ & $([0.3,0.56,0.89] ; 0.62,0.26)$ & $([0.3,0.57,0.9] ; 0.63,0.25)$ \\
\hline
\end{tabular}


Table 5. The ranking results of alternatives by the score function

\begin{tabular}{|c|c|c|c|}
\hline$A_{i}$ & TIFWA & TIFOWA & TIFHA \\
\hline$H\left(\tilde{r}_{1}\right)$ & 0.075 & 0.087 & 0.098 \\
\hline$H\left(\tilde{r}_{2}\right)$ & 0.105 & 0.105 & 0.091 \\
\hline$H\left(\tilde{r}_{3}\right)$ & 0.112 & 0.107 & 0.107 \\
\hline$H\left(\tilde{r}_{4}\right)$ & 0.140 & 0.142 & 0.153 \\
\hline & $A_{4} \succ A_{3} \succ A_{2} \succ A_{1}$ & $A_{4} \succ A_{3} \succ A_{2} \succ A_{1}$ & $A_{4} \succ A_{3} \succ A_{1} \succ A_{2}$ \\
\hline
\end{tabular}

From the table 5 we can see that the best alternatives is $A_{4}$.Therefore, the method proposed in this paper is reasonable and feasible.

\section{Conclusion}

In this paper investigates the MCDM problem, in which the criteria values are in the form of TIFNs, and a new MCDM method is proposed. Based on the expected values, the score function and the accuracy function of triangular intuitionistic fuzzy number are given, the method for ranking triangular intuitionistic fuzzy numbers are developed. The distance between triangular intuitionistic fuzzy numbers is defined. Then, the concept of the TIFWG, the TIFOWG and TIFHG operators has been defined, which extends the weighted geometric (WG) operator, the ordered weighted geometric (OWG) operator and the hybrid geometric (HG) operator to accommodate the situations where the decision information are triangular intuitionistic fuzzy numbers. The novelty of the TIFHG operator is that it reflects the importance degrees of both the given argument and the ordered position of the argument and can relieve the influence of unfair arguments. Furthermore, maximizing deviation method is used to determine the criteria weight, and we have developed an approach to multi-criteria decision making with triangular intuitionistic fuzzy information based on the TIFWG operator, the TIFOWG operator, and the TIFHG operator. Finally, an illustrative example is given to verify the developed approach and to demonstrate its practicality and effectiveness. Therefore, the geometric average operators of TIFNs greatly enrich the research content of IF MCDM and provide a new tool of information fusion for solving decision problems under IF environments. In the future, we shall continue working in the extension and application of the developed operators and decision making method to other domains.

\section{Acknowledgments}

This work is supported by the National Natural Science Foundation of China (No. 71331002, NO.71271072 and NO.71201145), the Doctoral Foundation of Ministry of Education of China (No. 20110111110006). The authors also are very grateful to the anonymous reviewers and editors for their very valuable comments and suggestions. 


\section{References}

[1] Atanassov K. Remark on intuitionistic fuzzy numbers. Notes on intuitionistic fuzzy sets, 13, 2007, 29-32.

[2] Atanassov K. Remark on operations "subtraction" over intuitionistic fuzzy sets. Notes on intuitionistic fuzzy sets, 15, 2009, 24-9.

[3] Atanassov K. A New Approach to the Distances between Intuitionistic Fuzzy Sets [M]. Information Processing and Management of Uncertainty in Knowledge-Based Systems Theory and Methods. Springer. 2010: 581-90.

[4] Atanassov K. On a new approach towards defining intuitionistic fuzzy subtractions. ACTA UNIVERSITATIS MATTHIAE BELII, series MATHEMATICS, 19, 2011, 11-20.

[5] Atanassov K., Vassilev P., Tcvetkov R. Intuitionistic Fuzzy Sets, Measures and Integrals. Sofia: "Prof. M. Drinov" Academic Publishing House, 2013.

[6] Atanassov K.T. Intuitionistic fuzzy sets. Fuzzy Sets and Systems, 20, 1986, 87-96.

[7] Atanassov K.T. A theorem for basis operators over intuitionistic fuzzy sets. Mathware \& soft computing, 8, 2008, 21-30.

[8] Atanassov K.T. New Intuitionistic Fuzzy Operations [M]. On Intuitionistic Fuzzy Sets Theory. Springer. 2012: 195-257.

[9] Boran F.E., Genç S., Kurt M., et.al. A multi-criteria intuitionistic fuzzy group decision making for supplier selection with TOPSIS method. Expert Systems with Applications, 36, 2009, 11363-8.

[10]Burillo P., Bustince H., Mohedano V. Some definitions of intuitionistic fuzzy number. First properties; The Proceedings of the 1st Workshop on Fuzzy Based Expert Systems, 1994.

[11]Chen Y., Li B. Dynamic multi-attribute decision making model based on triangular intuitionistic fuzzy numbers. Scientia Iranica, 18, 2011, 268-74.

[12]Farhadinia B. A theoretical development on the entropy of interval-valued fuzzy sets based on the intuitionistic distance and its relationship with similarity measure. Knowledge-Based Systems, 39, 2013, 79-84.

[13]Hwang C.-M., Yang M.-S., Hung W.-L., et.al. A similarity measure of intuitionistic fuzzy sets based on the Sugeno integral with its application to pattern recognition. Information Sciences, 189, 2012, 93-109.

[14]Jianqiang W., Zhong Z. Aggregation operators on intuitionistic trapezoidal fuzzy number and its application to multi-criteria decision making problems. Systems Engineering and Electronics, Journal of, 20, 2009, 321-6.

[15]K. A., G. G. Interval valued intuitionistic fuzzy sets. Fuzzy Sets and Systems, 31, 1989, 343-9.

[16]Li D.-F. A note on "using intuitionistic fuzzy sets for fault-tree analysis on printed circuit board assembly". Microelectronics Reliability, 48, 2008, 1741.

[17]Li D.-F. A ratio ranking method of triangular intuitionistic fuzzy numbers and its application to MADM problems. Computers \& Mathematics with Applications, 60, 2010, 1557-70.

[18]Li D.F., Nan J.X., Zhang M.J. A ranking method of triangular intuitionistic fuzzy numbers and application to decision making. International Journal of Computational Intelligence Systems, 3, 2010, 522-30.

[19]Liu P., Zhang X. Research on the supplier selection of a supply chain based on entropy 
weight and improved ELECTRE-III method. International Journal of Production Research, 49, 2011, 637-46.

[20]Nan J.-X., Li D.-F., Zhang M.-J. A lexicographic method for matrix games with payoffs of triangular intuitionistic fuzzy numbers. International Journal of Computational Intelligence Systems, 3, 2010, 280-9.

[21]Robinson P.J., Amirtharaje C.H. Extended TOPSIS with Correlation Coefficient of Triangular Intuitionistic Fuzzy Sets for Multiple Attribute Group Decision Making. International Journal of Decision Support System Technology (IJDSST), 3, 2011, $15-41$.

[22]Shu M.-H., Cheng C.-H., Chang J.-R. Using intuitionistic fuzzy sets for fault-tree analysis on printed circuit board assembly. Microelectronics Reliability, 46, 2006, 2139-48.

[23]Tan C. A multi-criteria interval-valued intuitionistic fuzzy group decision making with Choquet integral-based TOPSIS. Expert Systems with Applications, 38, 2011, 3023-33.

[24]Tcvetkov R., Szmidt E., Kacprzyk J., et.al. A modified Hausdorff distance between intuitionistic fuzzy sets. COMPTES RENDUS DE L ACADEMIE BULGARE DES SCIENCES, 65, 2012, 1035-42.

[25]Wan S.-P. Survey on intuitionistic fuzzy multi-attribute decision making approach. Control and decision, 25, 2010, 1601-6.

[26]Wan S.-P., Li D.-F. Possibility mean and variance based method for multi-attribute decision making with triangular intuitionistic fuzzy numbers. Journal of Intelligent and Fuzzy Systems, 24, 2013, 743-54.

[27]Wan S.-P., Wang Q.-Y., Dong J.-Y. The extended VIKOR method for multi-attribute group decision making with triangular intuitionistic fuzzy numbers. Knowledge-Based Systems, 52, 2013, 65-77.

[28]Wang J.-Q., Nie R., Zhang H.-Y., et.al. New operators on triangular intuitionistic fuzzy numbers and their applications in system fault analysis. Information Sciences, 251, 2013, 79-95.

[29] Wang J.-Q., Zhang H.-Y. Multicriteria decision-making approach based on Atanassov's intuitionistic fuzzy sets with incomplete certain information on weights. Fuzzy Systems, IEEE Transactions on, 21, 2013, 510-5.

[30]Wang J., Zhang Z. Multi-criteria decision-making method with incomplete certain information based on intuitionistic fuzzy number. Control and decision, 24, 2009, 226-30.

[31]Wang Y. Using the method of maximizing deviations to make decision for multi-indices. System Engineering and Electronics, 7, 1998, 24-6.

[32]Wei G.-W. Maximizing deviation method for multiple attribute decision making in intuitionistic fuzzy setting. Knowledge-Based Systems, 21, 2008, 833-6.

[33]Wei G.-W. GRA method for multiple attribute decision making with incomplete weight information in intuitionistic fuzzy setting. Knowledge-Based Systems, 23, 2010, 243-7.

[34]Wei G. Some Arithmetic Aggregation Operators with Intuitionistic Trapezoidal Fuzzy Numbers and Their Application to Group Decision Making. Journal of Computers, 5, 2010, 345-51.

[35] Wu Z., Chen Y. The maximizing deviation method for group multiple attribute decision making under linguistic environment. Fuzzy Sets and Systems, 158, 2007, 1608-17.

[36]Xu Z. Intuitionistic fuzzy aggregation operators. Fuzzy Systems, IEEE Transactions on, 15, 2007, 1179-87. 
[37]Xu Z. Approaches to multiple attribute group decision making based on intuitionistic fuzzy power aggregation operators. Knowledge-Based Systems, 24, 2011, 749-60.

[38]Xu Z., Chen J. On geometric aggregation over interval-valued intuitionistic fuzzy information; The Fourth International Conference on Fuzzy Systems and Knowledge Discovery(FSKD 2007) Haikou, China, 2007.

[39]Xu Z., Yager R.R. Some geometric aggregation operators based on intuitionistic fuzzy sets. International Journal of General Systems, 35, 2006, 417-33.

[40]Zadeh L.A. Fuzzy sets. Information and control, 8, 1965, 338-53.

[41]Zhang S.-F., Liu S.-Y. A GRA-based intuitionistic fuzzy multi-criteria group decision making method for personnel selection. Expert Systems with Applications, 38, 2011, 11401-5.

Received March, 2014 